\title{
Rapid Response of Confluent Reticulated Papillomatosis to the Combination of Doxycycline and Topical Tretinoin: A Case Report
}

Kutlu $\mathbf{0}^{1 *}$, Özdemir $\mathrm{P}^{2}$, Karadeniz $\mathrm{TB}^{3}$, Ünverdi $\mathrm{H}^{4}$, Şahin $\mathrm{M}^{5}$, Kutlu FN $^{6}$ and Ekşioğlu $\mathrm{HIM}^{7}$

${ }^{1}$ Develi Hatice Muammer Kocatürk State Hospital, Department of Dermatology and Venereology, Turkey

${ }^{2}$ Nazilli State Hospital, Department of Dermatology and Venereology, Turkey

${ }^{3}$ Kırıkkale Yüksek İhtisas State Hospital, Department of Dermatology and Venereology, Turkey

${ }^{4}$ Dr.Ersin Arslan Training and Research Hospital, Department of Pathology, Turkey

${ }^{5}$ Kudret International Hospital, Department of Dermatology and Venereology, Turkey

${ }^{6}$ Health Sciences University, Kayseri City Hospital, Department of Dermatology and Venereology, Turkey

${ }^{7}$ Health Sciences University Ankara Health Training and Research Center, Department of Dermatology and Venereology, Turkey

*Corresponding author: Ömer Kutlu, MD, Develi Hatice Muammer Kocatürk State Hospital, Department of Dermatology and Venereology, Kayseri, Turkey, Tel: +90 054577136 83; Fax: 035262162 23; Email: omerkutlu22@gmail.com

\section{Abstract}

Confluent reticulated papillomatosis(CRP) is a disease which is characterized with peripheral, reticular hyperpigmented patches and hyperkeratotic papules, first defined in 1927. The disease, also known as Gougerot Carteaud syndrome, mostly affects young women in 2nd and 3rd decade of life. Interscapular, epigastric and intermammary areas are involved in most of the cases. Although etiopathogenesis of the disease is still unclear, antibiotics such as minocycline, doxycycline, azithromycin and retinoic acid derivatives are being used in the treatment. Clearance of the lesion in CRP may take a long time. Herein, we present our case in order to emphasize rapid response to the combination therapy of systemic doxycycline and topical tretinoin.

Keywords: Confluent reticulated papillomatosis; Doxycycline; Tretinoin 


\section{Introduction}

Confluent reticular papillomatosis (CRP) was first described by Gougerot-Carteaud in 1927 as a disease in which peripheral reticular hyperpigmented patches with hyperkeratotic papules were observed $[1,2]$. CRP is often seen in the 2nd and 3rd decade especially in young women. Lesions mostly involve interscapular, epigastric and intermamarian areas. Acanthosis nigricans and pityriasis versicolor are frequently included in the differential diagnosis of the disease. Hyperkeratosis, papillomatosis, diminished granular layer and mild acanthosis are among the histopathological findings of CRP $[3,4]$. Antimicrobial agents such as doxycycline, azithromycin and retinoic acid derivatives are used in the treatment of the disease $[5,6]$. Herein, we present a young female patient who responded rapidly to the combined treatment of systemic doxycycline and topical tretinoin.

\section{Case Report}

A 23-year-old female patient presented with a brown color change and a thickening of the skin on the mid-chest, which began 6 months ago. She had a history of abdominal obesity and menstrual cycle irregularities. Her family history had no similar disease. System examination and physical examination were unremarkable. Dermatological examination revealed multiple brown papules and a few macules in the intermammary region. It was seen that a large part of these papules came together to form a velvety plaque of $5 \times 8 \mathrm{~cm}$ (Figure 1). Her routine hematological and biochemical tests were within normal limits. Insulin resistance and other hormonal abnormalities were not detected in the patient. Syphilis, HIV, hepatitis serologies and autoimmune panel were negative. Punch biopsy was performed with preliminary diagnoses of pityriasis versicolor, prurigo pigmentosa, amyloidosis and confluent reticular papillomatosis. Histopathological examination of this biopsy specimen showed orthokeratotic hyperkeratosis in the epidermis, papilllamatous, mild pigmentation in the basal epidermis, mild perivascular lymphocyte exocytosis in the superficial vascular plexus and diffuse melanophages (Figure 2). Based on these clinical and histopathological findings, diagnosis of CRP was made. Doxycycline $100 \mathrm{mg} /$ day orally and once daily application of topical tretinoin was started. After 20 days of follow-up, the lesion was clinically almost completely recovered (Figure 3 ).
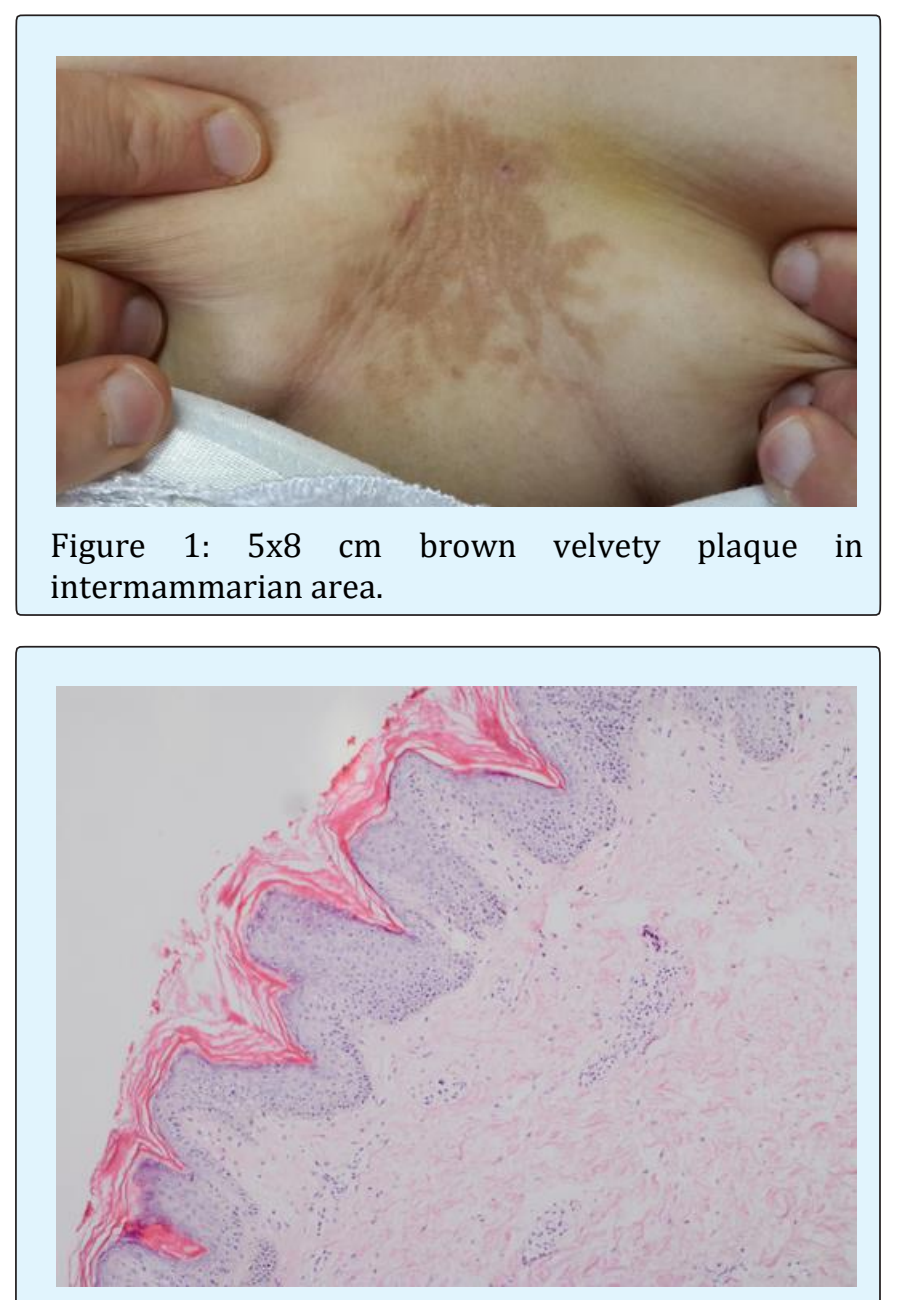

Figure 2: Epidermis with marked papillomatous and hyperkeratosis signs HEx100.

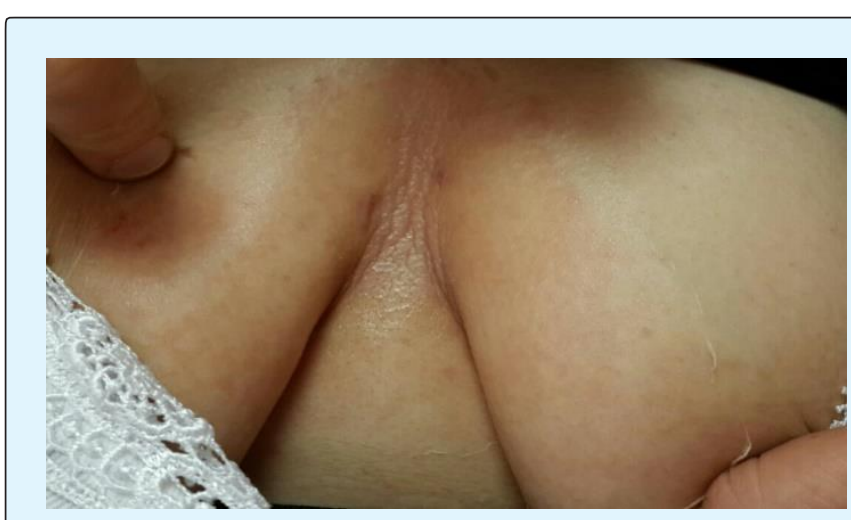

Figure 3: $20^{\text {th }}$ day of the treatment with doxycycline capsule $100 \mathrm{mg} /$ day combined with daily topical retinoic acid. 


\section{Clinical Dermatology Open Access Journal}

\section{Discussion}

Confluent reticular papillomatosis was first defined by Gougerot-Carteaud as a disease in which hyperkeratotic papules and peripheral reticular hyperpigmented patches were observed [1]. The disease is usually sporadic and rarely seen. It occurs mostly in young adults [4]. Davis, et al. proposed diagnostic criteria of CRP. These criteria include: (i) the presence of brown macules or patches containing at least part of the reticular and papillomatosis structure (ii) upper trunk and neck involvement (iii) negative fungal examination (iv) failure to respond to antifungal therapy; and (v) good response to the treatment of minocycline [3]. Although the etiopathogenesis of the disease is not known, endocrinological conditions such as Type 2 diabetes mellitus, Cushing syndrome, menstrual abnormalities, thyroid dysfunction, hirsutism and obesity may be associated with the disease [2,3]. In this case, the presence of a few macules concomitant with brown papillomatosis plaque involving trunk and negative periodic acid-Schiff (PAS) staining for the fungus in the biopsy was compliant with criteria defined by Davis et al. Furthermore, the body mass index of the patient was between 30-35 with a class 1 obese. Previous studies in the literature, it has been showed that obesity may be associated with CRP [7]. Obesity and diabetes mellitus are common in individuals with CRP, Therefore, it can be speculated that insulin resistance may play a role in the etiology of the disease.

Acanthosis nigricans and pityriasis versicolor are the most common differential diagnosis of confluent reticular papillomatosis. Differentiation between tinea versicolor and CRP can be done by native examination. On the other hand, differentiation between acanthosis nigricans and CRP is difficult. Some of the authors propose that acanthosis nigricans and CRP are in the same spectrum due to their clinical and histopathological similarities [8]. It can be considered that these two diseases may be the same entity due to the fact that similar clinical and histopatological features of the achantosis nigrikans and CRP besides their association with similar endocrinological diseases. As in our case, hyperkeratosis, papillomatosis, acanthosis and increased melanin accumulation are observed in the histopathology of CRP.

Minocycline is one of the most effective agents in the treatment of the disease $[5,9,10]$. However, oral antibiotics such as azithromycin, erythromycin and retinoic acid derivatives have been used to treat the disease at varying success rates $[6,11,12]$.
It can take several months until total clearance of the lesions occurs [13-15]. Doxycycline 1x100 mg/day orally and once daily application of topical retinoic acid were commenced as treatment. On the 20th day of treatment, the patient's lesions improved significantly. In this context, it can be considered that in a patient with CRP, especially those with prominent papillomatous structures and localized involvement may respond better to the combined oral doxycycline and topical retinoic acid treatment in terms of clearance time.

In a report published by Seongmoon Jo et al., in which only 2x100 mg oral doxycycline treatment was given, CRP lesions were completely recovered in 2 months [15]. In addition, when the literature is reviewed, the complete recovery time of the patients with CRP who received only topical retinoid or oral minocycline extended from 2 months to 1 year. Moreover, it was reported that a patient with CRP had rapid response to the treatment of oral minocycline combined with tazarotene $[3,7,14,16]$. Effective combination treatment with minocycline/doxycycline can be done more financially with topical tretinoin instead of tazarotene especially in the developing countries.

\section{Conclusion}

CRP may be misdiagnosed as prurigo pigmentosa, pityriasis versicolor and amyloidosis since it clinically appears as hyperpigmented and hyperkeratotic lesions. Therefore, suspected cases require a biopsy. Antibiotics such as minocycline, doxycycline and retinoic acids, vitamin d derivatives have been used in the treatment of disease with varying response rates. As can be understood from this case, the combination of topical tretinoin with oral doxycycline therapy may lead to a rapid response to the treatment.

\section{References}

1. Gougerot H, Carteaud A (1927) Papillomatose pigmentee innominee. Bull Soc Fr Dermatol Syphiligr 34: 719-721.

2. Ferreira LM, Diniz LM, Ferreira CJ (2009) Confluent and reticulated papillomatosis of Gougerot and Carteaud: Report of three cases. An Bras Dermatol 84(1): 78-81.

3. Davis MD, Weenig RH, Camilleri MJ (2006) Confluent and reticulate papillomatosis (Gougerot-Carteaud syndrome): A minocycline-responsive dermatosis 


\section{Clinical Dermatology Open Access Journal}

without evidence for yeast in pathogenesis. A study of 39 patients and a proposal of diagnostic criteria. $\mathrm{Br} \mathrm{J}$ Dermatol 154(2): 287-293.

4. Scheinfeld N (2006) Confluent and reticulated papillomatosis: A review of the literature. Am J Clin Dermatol 7(5): 305-313.

5. Yamamoto Y, Kadota M, Nishimura Y (2006) A Case of maxacalcitol-resistant confluent and reticulated papillomatosis successfully treated with minocycline. J Dermatol 33(3): 223-224.

6. Jang HS, Oh CK, Cha JH, Cho SH, Kwon KS (2001) Six cases of confluent and reticulated papillomatosis alleviated by various antibiotics. J Am Acad Dermatol 44(4): 652-655.

7. Filho FB, Quaresma MV, Rezende FC, Kac BK, Nery JA, et al. (2014) Confluent and reticulate papillomatosis of Gougerot-Carteaud and obesity: Dermoscopic findings. Anais Brasileiros de Dermatologia 89(3): 507-509.

8. Cockerell CJ, Larsen F (2008) Confluent and reticulated papillomatosis. In: Bolognia JL, Jorizzo JL, Rapini RP, (Eds.), Dermatology. $2^{\text {nd }}$ (Edn.), New York: Mosby Elsevier, pp: 1677-1678.

9. Poskitt L, Wilkinson JD (1993) Clearance of confluent and reticulate papillomatosis of Gougerot and Carteaud with minocycline. Br J Dermatol 129(3): 351-353.
10. Puig L, de Moragas JM (1995) Confluent and reticulated papillomatosis of Gougerot and Carteaud: minocycline deserves trial before etretinate. Arch Dermatol 131(1): 109-110.

11. Lee MP, Stiller MJ, McClain SA, Shupack JL, Cohen DE (1994) Confluent and reticulated papillomatosis: response to high-dose oral isotretinoin therapy and reassessment of epidemiologic Data. J Am Acad Dermatol 31(2): 327-331.

12. Schwartzberg JB, Schwartzberg HA (2000) Response of confluent and reticulate papillomatosis of Gougerot and Carteaud to topical tretinoin. Cutis 66(4): 291293.

13. Carlin N, Marcus L, Carlin R (2010) GougerotCarteaud Syndrome treated with 13-cis-retinoic Acid. J Clin Aesthetic Dermatol 3(7): 56-57.

14. Tseng HW, Tseng HH, Wu CS (2013) Confluent and reticulate papillomatosis treated with minocycline and tazarotene. Cutis 91(4): 194-197.

15. Jo S, Park HS, Cho S, Yoon HS (2014) Updated diagnosis criteria for confluent and reticulated papillomatosis: a case report. Annals of Dermatology 26(3): 409-410.

16. Hudacek KD, Haque MS, Hochberg AL, Cusack CA, Chung CL (2012) An unusual variant of confluent and reticulated papillomatosis masquerading as tinea versicolor. Arch Dermatol 148(4): 505-508. 